Z. Klin. Chem. Klin. Biochem.

13. Jg. 1975 , S. $429-435$

\title{
Über neue Proteaseinhibitoren mit breiter Wirkungsspezifität bei dem Polychaeten Sabellastarte indica (Savingny), I. Mitteilung
}

\author{
Von C. Gauwerky, G. Corman und G. Uhlenbruck \\ Aus der Abteilung Experimentelle Innere Medizin der 1. Medizinischen Universitätsklinik Köln
}

(Eingegangen am 17. Februar/10. Juni 1975)

Zusammenfassung: Es wird über neue bisher unbekannte Proteaseinhibitoren in den Tentakeln des Anneliden Sabellastarte indica Savingny berichtet. Hierbei handelt es sich um vernutlich bis zu fünf Isoinhibitoren, die elektrophoretisch voneinander getrennt werden. Sie besitzen Hemmaktivitäten gegenüber Trypsin, Plasmin, Chymotrypsin und Kallikrein. Von den herkömmlichen Proteaseinhibitoren, die bisher bei Invertebraten gefunden wurden, unterscheidet sich unser Inhibitormaterial hinsichtlich des hohen Molekulargewichtes, seines empfindlichen Verhaltens bei Temperaturbelastung und seines isoelektrischen Punktes, der in den schwach sauren Bereich fällt. Andererseits weist er gewisse Gemeinsamkeiten mit dem Kunitz-Inhibitor aus Sojabohne und dem Ovomucoid auf. Eine mögliche Bedeutung hinsichtlich der Beeinflussung des fibrinolytischen Systems wird diskutiert.

\section{New protease-inhibitors with broad specificity in the polychaet sabellastarte indica (Savingny), I.}

Summary: In this paper we describe new and so far unknown protease inhibitors present in the tentacles of the annelid Sabellastarte indica Savingny. At least five different isoinhibitors with inhibitory activity towards trypsin, plasmin, chymotrypsin and kallikrein can be separated electrophoretically. Our protease inhibitor active material differs from the other well known protease inhibitors found in invertebrates in its high molecular weight, in that it is heat-labile and in the occurrence of the isoelectric point in the weakly acid region.

On the other hand, the new protease inhibitors have some similarities to the soybean inhibitor described by Kunitz, and to ovomucoid.

We also discuss the possibility that these inhibitors may influence the fibrinolytic system.

\section{Einführung}

Enzyminhibierende Systeme bei niederen Tieren, insbesondere bei Blutegeln und Darmparasiten sind seit Ende des letzten Jahrhunderts bekannt. Dem Blutegel Hirudo medicinalis galt besonders praktisch-medizinisches Interesse, da bis zur Entdeckung des Heparins Blutegel-Extrakte die einzigen Mittel waren, die Blutgerinnung herabzusetzen. Inzwischen ist es Markwardt (1) gelungen, das hierfür verantwortliche Protein Hirudin rein darzustellen und Fritz (2) konnte von diesem "Antithrombin" die Trypsin/Plasmininhibitoren Bdellin $A$ und Bdellin B, einschließlich ihrer Untergruppen, abtrennen und charakterisieren. Dies war insofern von Interesse, als diese Inhibitoren Bdellin A und Bdellin B nicht geringe Anteile der käuflichen Hirudinpräparate ausmachten.
Der Nematode Ascaris lumbricoides zog um die Jahrhundertwende vor allem das Augemmerk der Physiologen auf sich, da es einer Erklärung bedurfte, wie dieser Parasit imstande war, im Verdauungstrakt eines Wirtstieres zu überleben (siehe auch Frenzel (3)). Hervorragende Untersuchungen hierzu führte bereits Weinland 1903 (4) durch; er konnte sogenannte „Antifermente" für die Widerstandsfähigkeit der Nematoden gegen pankreatische Verdauungsenzyme verantwortlich machen. Wir wissen heute, besonders auf Grund der ausführlichen Beschreibung von R. J. Peanasky \& Ghaleb M. Abu-Erreish (5), daß im Ascaris-Extrakt vier Gruppen von Proteaseinhibitoren vorhanden sind: einmal der am längsten bekannte Chymotrypsininhibitor, der auch Subtilisin und Elastase hemmt, und über den Peanasky \& Laskowski Sr. (6) und Rhodes (7) ausfuihr- 
lich berichten. Nach Peanasky \& AbuErreish handelt es sich hierbei um vier diskelektrophoretisch unterscheidbare Isoinhibitoren. Der Trypsininhibitor-haltige Anteil, der sich elektrophoretisch vom Chymotrysininhibitor trennen läßt, setzt sich aus zwei Trypsininhibitoren zusammen, die verschiedentlich dargestellt und unterschiedlich charakterisiert wurden $(5,8,9)$. Die dritte Inhibitorengruppe ist gegen das Verdauungsenzym Pepsin gerichtet und setzt sich aus 4 Isoinhibitoren zusammen. Das vierte Enzym, das ebenfalls von $A$ scarisExtrakt gehemmt wird, ist Carboxypeptidase A (3 Isoinhibitoren) (9).

Auch bei Mollusken konnten Proteaseinhibitoren gefunden werden, und zwar in verschiedenen Organen. Werle et al. beschrieben 1958 einen Hemmkörper für Trypsin und Kallikrein in der Hämolymphe von Helix pomatia (10). Das gleiche stellte auch Kareem für die Hämolymphe von Achatina fulica fest (11). Cheesman machte bereits 1958 auf einen Proteaseinhibitor in den Eiern der Schnecke Pomacea canaliculata australis aufmerksam (12). Dieser Chromoinhibitor ist ein Glycoprotein, das schon damals gereinigt werden konnte. Die nähere Charakterisiening erfolgte durch Norden 1972 (13).

Unsere Kölner Arbeitsgruppe fand dann Proteaseinhibitoren bei anderen Schnecken, und zwar sowohl in den Eiern als auch in den Eiweißdrüsen des Geschlechtsapparates. Ein besonders starkes proteaseinhibitorisches Prinzip in der Eiweißdrüse von Helix pomatia wurde von Uhlenbruck $(14,15)$ entdeckt. Tschesche $(16)$ bestätigte diese Befunde kurz darauf und konnte über die Isolierung und Reindarstellung niedermolekularer Inhibitoren berichten. Diese Inhibitoren besitzen besonderes entwicklungsgeschichtliches Interesse, weil nämlich festgestellt werden konnte, daß die Aminosäurensequenzanalyse wesentliche Übereinstimmungen mit dem Trypsin-Kallikreininhibitor aus Rinderorganen (= Trasylol) ergab $(17,18)$.

Für einen weiteren Vertreter der Cephalopoden, nämlich Loligo vulgaris, konnte Tschesche (19) ebenfalls niedermolekulare Proteaseinhibitoren darstellen. Beress et al. (20) fanden Proteaseinhibitoren bei Anthozoa. Auch die Inhibitoren in den Tentakeln von Anemonia sulcata scheinen dem Trasylol sehr nahe verwandt zu sein (Fritz (21)): sie hemmen neben Trypsin, Chymotrypsin, Plasmin und Organkallikrein auch Serumkallikrein, so daß eine Ähnlichkeit in der Tertiärstruktur des reaktiven Zentrums offensichtlich ist.

Die physiologische Bedeutung dieser Inhibitoren liegt ganz eindeutig in einer Schutzfunktion gegenüber enzymatischen Einflüssen, sowohl exogenen als auch endogenen Ursprungs. Diese Schutzfunktion kommt aber auch den Agglutininen zu, so daß für Helix pomatia (22) mit dem starken Anti-A Agglutinin und dem hohen. Inhibitorgehalt in der Eiweißdrüse $(14,15)$ sogar ein doppelter „Abwehr"mechanismus existiert. Im Rahmen unserer Untersuchungen über derartige Defensivmechanismen konnten wir ein besonders starkes proteaseinhibitorisches Prinzip bei dem Anneliden Sabellastarte indica Savingny finden, welches ein klassisches Inhibitionsmuster gegen Trypsin, $\alpha$-Chymotrypsin, Plasmin und Kallikrein aufweist und dessen Darstellung und Charakterisierung uns lohnenswert erschien.

\section{Material}

Sabellastarte indica Sav. (tropischer Röhrenwurm) aus der Nähe von Singapur konnte käuflich erworben werden.

\section{Enzyme}

Trypsin (vom Schwein) krist. lyophilisiert (EC 3.4.21.4) und $\alpha$-Chymotrypsin (vom Rind) krist: lyophilisiert (EC 3.4.21.1) wurden von Merck bezogen. Plasmin (vom Schwein) $10 \mathrm{NE} / \mathrm{mg}$ (EC 3.4.21.7) und Subtilisin krist. 32,9 AE/g (EC 3.4.21.14) wurden von der NOVO-Industrie zur Verfügung gestellt. Kallikrein vom Schweinepankreas mit $12900 \mathrm{KE} / \mathrm{mg}$ (EC 3.4.21.8) erhielten wir von der Bayer AG, Wuppertal; Alkohol: NADoxidoreductase (EC 1.1.1.1) wurde von Boehringer/Mannheim käuflich erworben. Thrombin (EC 3.4.21.5) wurde als Topostasin von LaRoche benutzt.

\section{Substrate}

$N$-Benzoyl- $L$-arginin-äthylester, N-Benzoyl- $L$-arginin-p-nitroanilid, $N$-(3-Carboxypropionyl)- $L$-phenylalanin- $p$-nitroanilid und NAD als freie Säure erwarben wir von der Firma Merck, Azocasein von der Fa. Serva. Eichsubstanzen zur Molekulargewichtsbestimmung bezogen wir von der $\mathrm{Fa}$. Serva.

Zur Fibrinagarelektrophorese benutzten wir Fibrinogen vom Rind von den Behringwerken Marburg.

\section{Methoden}

Aufarbeitung des Rohex traktes

Sabellastarte indica wurde von der äußeren Mantelhülle befreit und der Tentakelkranz vom Körper getrennt. Aus den Tentakeln wurde ein wäßriger Extrakt bereitet, der 30 min bei $26000 \mathrm{~g}$ zentrifugiert, 24 Stunden gegen dest. Wasser dialysiert und anschließend lyophilisiert wurde. Von diesem Ausgangsmaterial wurde eine 1 proz. wäßrige Lösung angesetżt, die wir als „Rohextrakt 1\%“ bezeichnen und die das Ausgangsmaterial für alle Untersuchungen war, die in dieser Arbeit beschriebèn werden.

\section{Eiweißbestimmung}

Zur Bestimmung der spezifischen Aktivität des Inhibitormaterials (mIU/mg Eiweiß) führten wir Eiweißbestimmungen nach Lowry mit dem Folin-Ciocalteau-Reagenz durch (23).

\section{Enzymatische Bestimmungen}

Die Bestimmung der enzymatischen Aktivitäten von Enzymen bzw. die Ermittlung ihrer Hemmung durch den 1 proz. Rohextrakt von Sabellastarte indica wurde nach den allgemein üblichen Methoden (24) durchgeführt: für Trypsin, Plasmin und Thrombin diente N-Benzoyl- $L$-arginin- $p$-nitroanilid als Substrat, für Chymotrypsin $\mathrm{N}$-(3-Carboxypropionyl)- $L$-phenylalanin- $p$ nitroanilid, für Subtilisin Azocasein (25). Kallikrein wurde mit Hilfe des Umsatzes von NAD-Oxidoreductase bestimmt (26).

\section{Temporäres Hem mverhalten}

Die zur Erreichung des Hemmgleichgewichtes nötige Vorinkubationszzeit wurde wie folgt ermittelt: die für einen Testansatz günstige Enzy mmenge wurde mit so viel Inhibitor-Rohmaterial, wie zur 50proz. Hemmung der vorgelegten Enzym- 
menge nötig war, bci $25^{\circ} \mathrm{C}$ in geeigneter Pufferlösung inkubiert. Nach 5, 10, 15, 20, 30 Minuten, 1, 2, 4, 6, 12 und 24 Stunden versetzten wir diesen Inhibitor-Enzym-Ansatz mit der dem Enzym entsprechenden Substratlösung und bestimmten so die noch wirksame Inhibitormenge.

\section{Fibrinagarelektrophorese}

Zur Lokalisation des Inhibitors im elektrischen Feld führten wir die Fibrinagarelektrophorese in Anlehnung an die Beschreibung von Heimburger \& Schwick (27) durch. Die elektrophoretische Auftrennung erfolgte über 150 Minuten bei 150 Volt und $10 \mathrm{~mA}$ pro Objektträger. Die Ergebnisse konnten etwa 4 Stunden nach Diffusion der Enzymlösungen bei Raumtemperatur photographisch festgehalten werden.

\section{Molekulargewich tsbestimmung}

Die Molekulargewichtsbestimmung führten wir nach Andrews (28) mit Hilfe der Gelfiltration durch. Als Eichsubstanzen dienten DNP-Alanin, Cytochrom c, Chymotrypsinogen, Ovalbumin, Albumin aus Rinderserum und Aldolase in elnem Flüssigkeitsvolumen von $3 \mathrm{ml}$. Die inhibitorhaltige Testsubstanz wurde unter identischen Bedingungen chromatographiert und das Volumen der zur Elution des Inhibitors nötigen $\mathrm{NaCl}$ Lösung mit Hilfe von Enzymbestimmungen ermittelt. Zusätzlich bestimmten wir das Molekulargewicht nach Felgenhauer (29) durch Disk-Elektrophorese in Acrylamidgel (5-15\%) bei $\mathrm{pH}=8,9$. Referenzsubstanz war Myoglobin.

\section{Ergebnisse}

\section{Verhalten gegenüber proteolytischen Enzymen}

\section{Trypsin}

Die Inhibitoraktivität für den Rohextrakt errechnet sich aus dem Wert, der zu 50\% Hemmung des Enzyms führt, zu $4499 \mathrm{mIU} / \mathrm{ml}^{1}$ ). Bei einem Eiweißgehalt von $0,468 \mathrm{mg} / \mathrm{ml}$ ergibt sich damit eine spezifische Inhibitoraktivität von $9610 \mathrm{mIU} / \mathrm{mg}$ Protein.

Die Titrationskurve ist in Abbildung 1 wiedergegeben.

Die Hemmung des Enzyms verläuft bis zu $88 \%$ Inhibierung linear. Hinsichtlich des zeitlichen Verhaltens der Enzymhemmung konnten wir durch Veränderung der Vorinkubationszeit, die wir zwischen 5 Minuten und 24 Stunden variierten, keine Abnahme der Inhibitorwirksamkeit feststellen.

\section{Chymotrypsin}

Die Ausgangsmenge für $\alpha$-Chymotrypsin lag zwischen 2 und $3 \mathrm{mU}$. Aus der Inhibitormenge, bei der 50\% Hemmung ereicht wird, errechnet sich eine Inhibitoraktivität von $60 \mathrm{mIU} / \mathrm{ml} 1$ proz. Rohextrakt und eine spezifische Inhibitoraktivität von $128 \mathrm{mIU} / \mathrm{mg}$ Protein. Die Titrationskurve, die in Abbildung 2 wiedergegeben ist, zeigt, daß die Hemmung des Enzyms bis zu $71 \%$ linear verläuft. Gegenüber Chymotrypsin besitzt der Inhibitor ebenfalls Sofortwirkung. Die Veränderung der Vorinkubationszeit zwischen 5 Minuten und 24 Stunden führt zu keiner Veränderung der Inhibitorwirksamkeit.

1) $\mathrm{mIU}=$ milli-Inhibitoreneinheiten. 1 IU entspricht dabei der Verminderung der Substratspaltung um $1 \mu \mathrm{mol} / \mathrm{min}$.

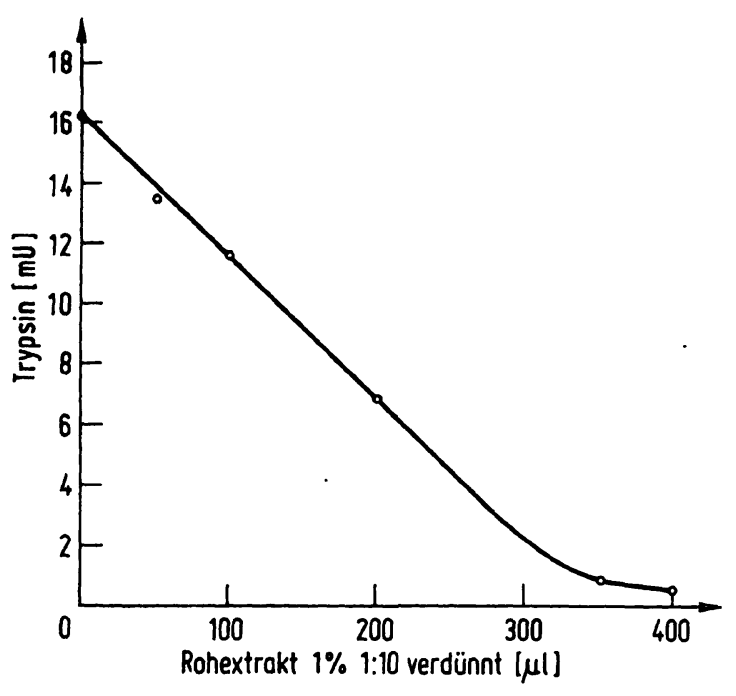

Abb. 1. Titration von Trypsin und Tentakel-Inhibitormaterial (1 proz. Rohextrakt). Vorgelegt wurden 16,2 mU Trypsin.

\section{Plasmin}

Vorgelegt wurden für jeden Versuchsansatz etwa $5 \mathrm{mU}$ Plasmin. Aus der Menge, bei der 50\% Hemmung ereicht wird, errechnet sich für den Rohextrakt eine Inhibitoraktivität von $136,9 \mathrm{mIU} / \mathrm{ml}$ oder eine spezifische Inhibitoraktivität von $292,5 \mathrm{mIU} / \mathrm{mg}$ Protein gegenüber Plasmin. Bis zu 72\% Hemmung verläuft die Hemmung des Enzyms linear (Abb. 2). Sie ist ebenfalls unabhängig von der Vorinkubationszeit, die wir zwischen 5 Minuten und 24 Stunden variierten.

\section{Kallikrein}

Die Kallikreinbestimmung im manuellen Verfahren mit Hilfe des NAD-Oxidoreductase-Umsatzes erlaubte eine Bestimmung der Inhibitoraktivität gegenüber SchweinePankreas-Kallikrein. Ausgangsmenge Kallikrein waren 6-8 mU. Da die Hemmung des Enzyms nur bis zu $60 \%$ Hemmung linear verläuft, beziehen sich unsere Angaben auf den Inhibitorwert, der bei 30\% Inhibition vorliegt. Es ergibt sich somit ein Inhibitorgehalt gegenüber

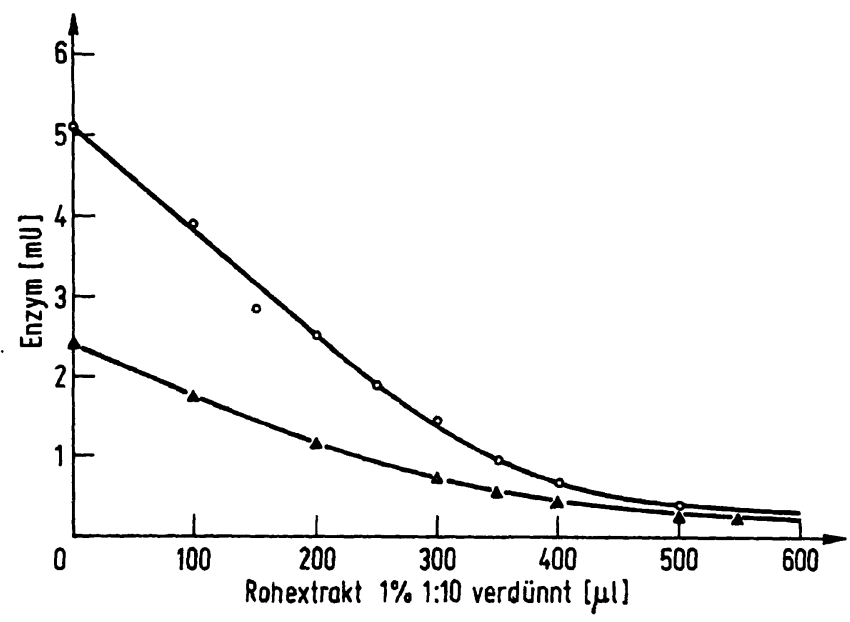

Abb. 2. Titration von Plasmin $\circ$ und Chymotrypsin $\triangle$ durch 1proz. Rohextrakt. 


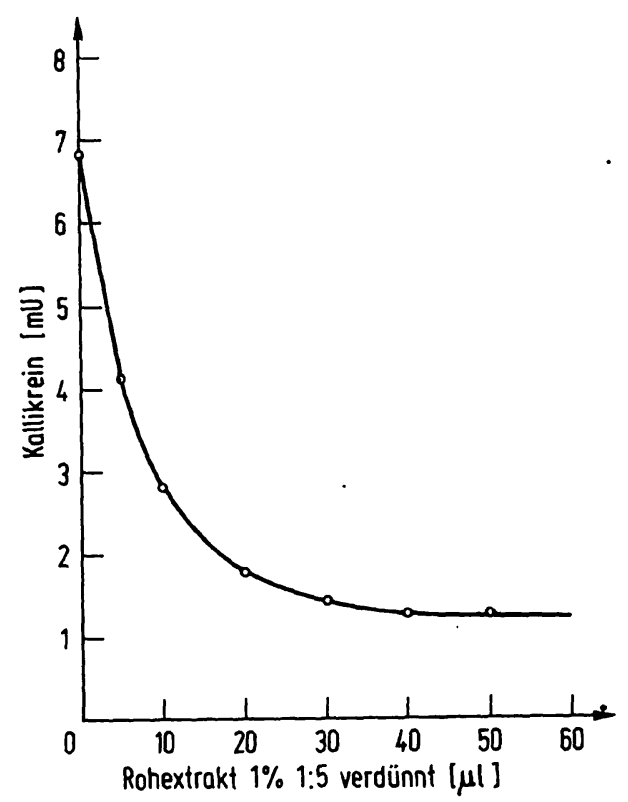

Abb. 3. Titration des Tentakel-Rohextraktes durch Kallikrein. Vorgelegt wurden etwa $7 \mathrm{mU}$ Kallikrein.

Schweine-Pankreas-Kallikrein von $2700 \mathrm{mIU} / \mathrm{ml}$ Rohextrakt und eine spezifische Inhibitoraktivität von $\mathbf{5 7 7 0}$ $\mathrm{mIU} / \mathrm{mg}$ Protein. Die Titrationskurve ist in Abbildung 3 wiedergegeben.

Die Veränderung der Vorinkubationszeit führte zu einer Veränderung der Inhibitoraktivität, derart, daß bei 30 Minuten Vorinkubationszeit das Inhibitormaterial maximale Wirksamkeit zeigt und von da an konstant bleibt.

\section{Subtilisin}

Es fand sich, daß durch Zugabe von 1 proz. Rohextrakt der Umsatz des Azocaseins durch Subtilisin nicht beeinflußt werden konnte.

\section{Thrombin}

Die Blockierung der Spaltung von N-Benzoyl- $L$-arginin$p$-nitroanilid durch Thrombin mit Tentakel-Rohmaterial wurde bei $\mathrm{pH}=8,4$ durchgeführt. Hierfür legten wir 120 NIH-Einheiten Thrombin vor. Der Substratumsatz wurde über 1 Stunde bei $405 \mathrm{~nm}$ beobachtet. Es zeigte sich, daß 0,1 ml 1 proz. Rohmaterial 23,16 NIH-Einheiten, $0,3 \mathrm{ml} 1$ proz. Rohmaterial 49,16 NIH Einheiten und $0,5 \mathrm{ml} 1$ proz. Rohmaterial 41,16 NIH-Einheiten zu hemmen in der Lage sind.

\section{Säure- und Thermostabilität}

1. Enzymbestimmungen konnten ergeben, daß die Inhibitoraktivität des 1 proz. Rohextraktes weder durch Citronensäurepuffer $\mathrm{pH}=2,2$ noch durch $0,014 \mathrm{~mol} / 1$ oder gar $0,08 \mathrm{~mol} / 1$ Perchlorsäure herabgesetzt wird. 2. Wird der 1 proz. Rohextrakt, unbehandelt oder in doppeltem Volumen $0,08 \mathrm{~mol} / 1$ Perchlorsäure, 30 Minuten steigenden Temperaturen ausgesetzt, ergibt sich eine Abhängigkeit, wie sie in Abbildung 4 dargestellt ist.

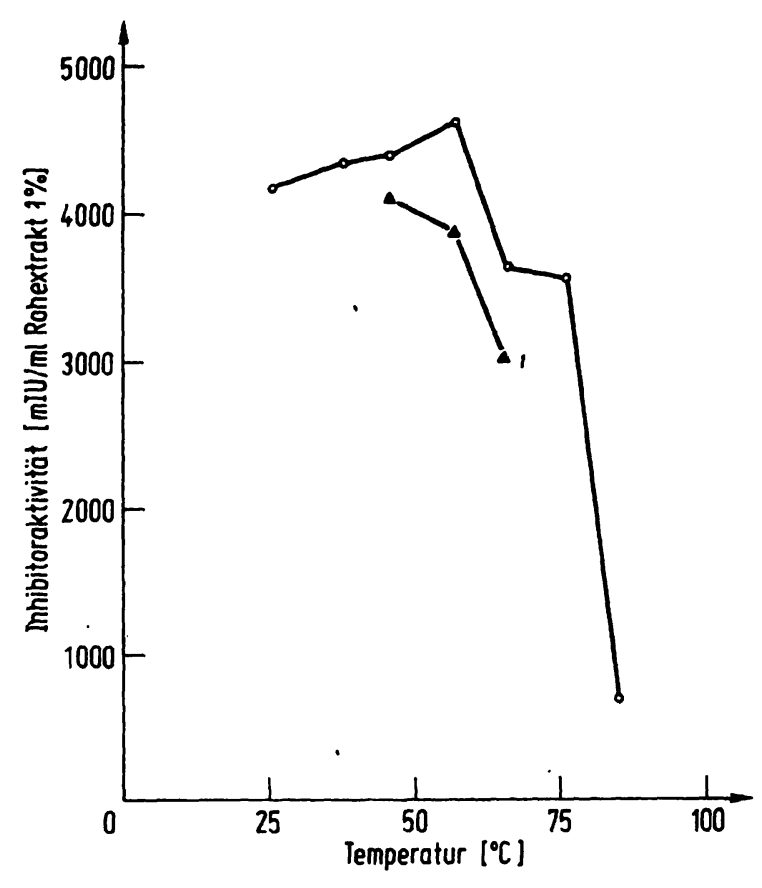

Abb. 4. Temperaturstabilität des unbehandelten 1proz. Rohextraktes 0 , und von Inhibitormaterial behandelt mit $0,08 \mathrm{~mol} / \mathrm{l}$ Perchlorsäure $\triangle$.

\section{Verhalten im elektrischen Feld}

Die Abbildung 5 macht deutlich, daß der Proteaseinhibitor, der die Fibrinverdauung durch Trypsin, Plasmin und Chymotrypsin verhindert, zur Anode wandert. Ebenfalls ist aus Abbildung 5 ersichtlich, daß zwei verschiedene Fibrinagarareale nicht verdaut wurden.
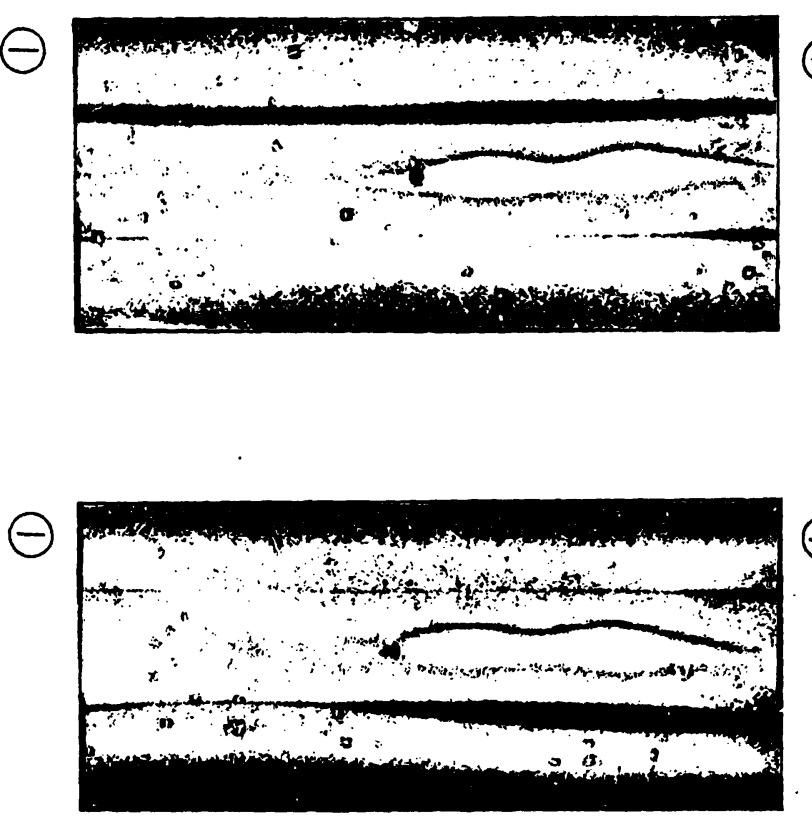

Abb. 5. Fibrinagarelektrophorese des 1proz. Rohextraktes; links Kathode, rechts Anode. oberer Graben jeweils Trypsinlösung unterer Graben a) Chymotrypsinlösung b) Plasminlösung 


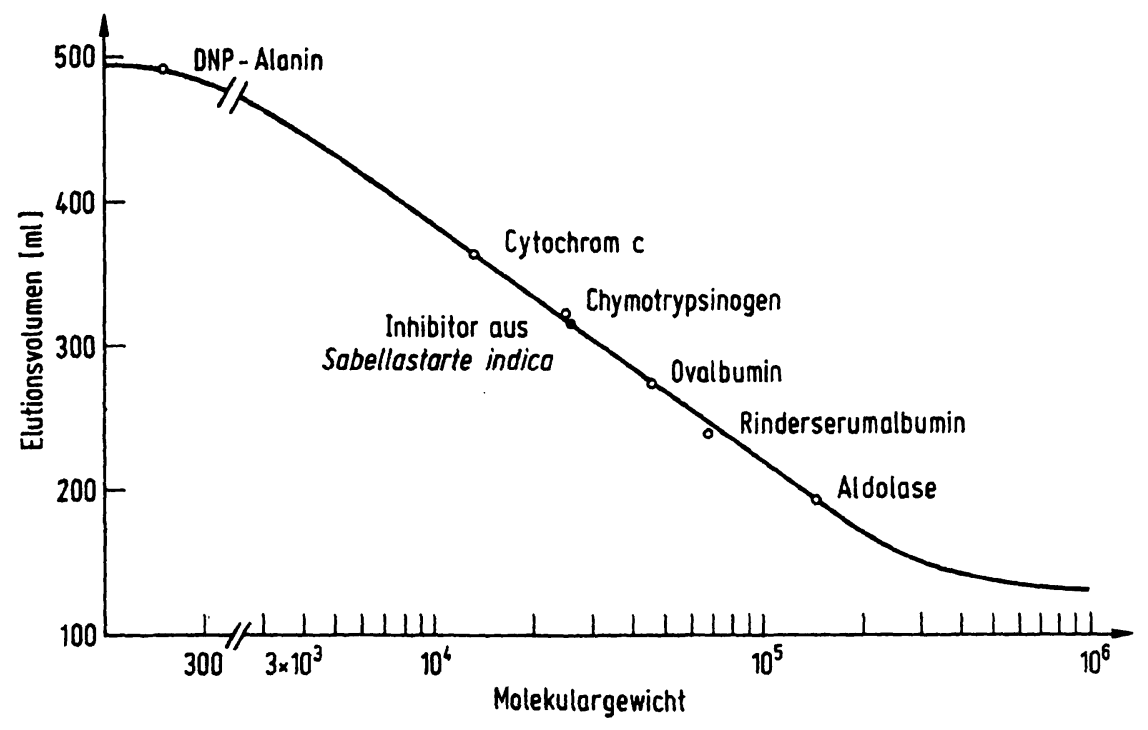

Abb. 6. Molekulargewich tsbestimmung mit Hilfe der Gelfiltration an Sephadex G 150 fine. Ermitteltes Molekulargewicht ungefähr 25500 (siehe Text).

\section{Molekulargewich tsbestim mung}

Die Ergebnisse der Molekulargewichtsbestimmung mit Hilfe der Säulenchromatographie brachten Ergebnisse, die in Abbildung 6 wiedergegeben sind. Nach dieser Methode wäre dem proteaseinhibitorischen Material im Rohextrakt ein Molekulargewicht von 25500 zuzuordnen. Mittels Disk-Elektrophorese konnten wir dieses Ergebnis besstätizen. (Abb. 7). Hierbei fand sich erstaunlicherweise, daß die fünf bei der Coomassiefärbung sich darstellenden Banden die selbe Ausschlußgrenze von $27 \%$ Acrylamidgel besitzen, was einem Molekulargewicht von 25100 entspricht. Zusätzliche Banden fanden sich nicht.

\section{Diskussion}

Die Proteaseinhibitoren in den Tentakeln des Anneliden Sabellastarte indica Sav. sind Vertreter für polyvalente Proteaseinhibitoren bei niederen Tieren, wie sie uns auch für Helix pomatia und Anemonia sulcata bekannt sind. Sie weisen das klassische Inhibitionsmuster auf mit Aktivitäten gegen Trypsin, Plasmin, Chymotrypsin und Kallikrein. Die Intensität der Inhibitorwirksamkeit gegenüber Trypsin mit einer spezifischen Aktivität von nahezu $10000 \mathrm{mIU} / \mathrm{mg}$ Protein ist erstaunlich und wäre am ehesten vergleichbar mit dem Inhibitor aus Samenblasen.

Weitere Untersuchungen werden klären müssen, ob es sich hierbei um eine Gruppe von Inhibitoren mit breitem Wirkungsspektrum handelt oder um mehrere Gruppen von Iso- oder Heteroinhibitoren. Mit Hilfe der Fibrinagarelektrophorese konnten wir bereits zeigen, daß im elektrischen Feld zwei anodisch liegende Inhibitorareale voneinander getrennt werden können. In der Disk-Elek- trophorese stellten sich fünf verschiedene Banden dar, die dasselbe Molekulargewicht von 25100 besitzen. Hiermit bestätigt sich einmal das Ergebnis der Gelfiltration, des weiteren läßt sich hieraus folgern, daß unser so bezeichnetes „Rohmaterial“ überraschend einheitlich ist und sich vermutlich aus bis zu 5 Isoinhibitoren zusammensetzt. Die vollständige Reindarstellung dieser Isoinhibitoren erfolgt deshalb zur Zeit.

Die meisten bekannten Proteaseinhibitoren besitzen ein Molekulargewicht unter 10000, haben basischen Charakter und zeichnen sich durch starke Säure- und Temperaturstabilität aus. Hochmolekulare Inhibitoren neben

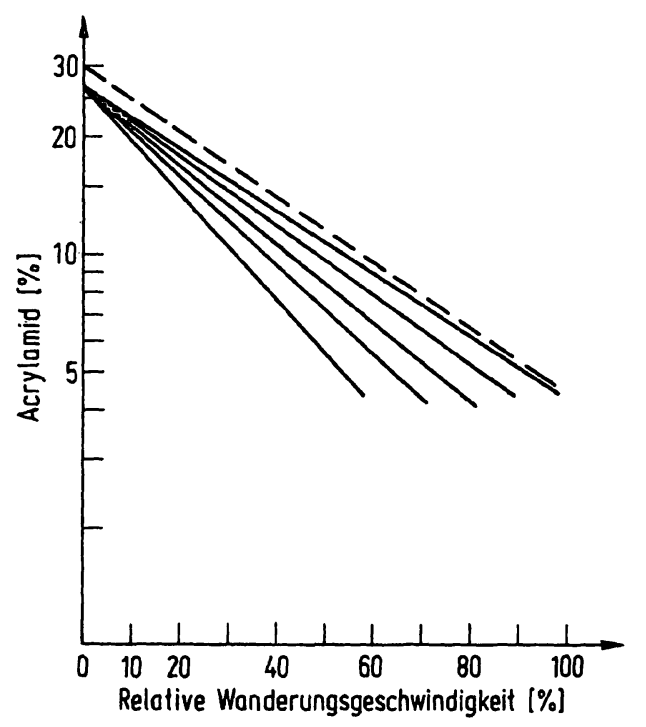

Abb. 7. Relative Wanderungsgeschwindigkeit gegen log der Acrylamid-Konzentration zur Bestimmung der Ausschlußgrenze.

- 5 sich darstellende Banden des Rohextraktes -..-Myoglobin. 
denen des Serums und dem Ovomucoid sind verhältnismäßig selten. Ein Molekulargewicht in der Größenordnung, wie wir es für unser Inhibitormaterial gefunden haben, besitzt auch der Kunitz- Inhibitor aus Sojabohnen (MG $=20$ 100) (30). Darüber hinaus besitzt auch dieser Trypsininhibitor die Eigenschaft, anodisch zu wandern (31). Arbeiten über den Sojabohnentrypsininhibitor sind sehr zahlreich erschienen, besonders um kinetische Studien und Untersuchungen über die Wechselwirkung von Enzym und Inhibitor darzustellen (32). Unser Inhibitor ist säurestabil, allerdings nicht, wenn er gleichzeitig über $56^{\circ} \mathrm{C}$ erhitzt wird - eine Eigenschaft, die dem Kunitz-Inhibitor ebenfalls zu kommt (33).

Hochmolekulare Proteaseinhibitoren sind in der Regel sekretorische Glycoproteine wie der Rindercolostruminhibitor, das Ovomucoid, der Ovoinhibitor oder hochmolekulare Inhibitoren aus der Eiweißdrüse der Weinbergschnecke (34). Das Molekulargewicht des Ovomucoid liegt bei 28000 , das des Ovoinhibitors bei 46000 (35). Auch unser Inhibitor dürfte ein Sekret sein, das sich in den Tentakeln befindet. Ebenfalls macht eine positive PAS-Färbung in der Disk-Elektrophorese wahrscheinlich, daß unser Inhibitor ein Glycoprotein ist.
Eine besondere Bedeutung haben Proteaseinhibitoren hinsichtlich ihrer Eigenschaft als Plasmininhibitoren in Bezug auf die Fibrinolyse gewonnen. So veranlaßte die starke plasminhemmende Wirkung des Trasylol 1956 Marx \& Werle dazu, diesen Inhibitor als Therapeuticum zur Hyperfibrinolyse vorzuschlagen (36). Ferner ist vom Sojabohneninhibițor bekannt, daß die proteolytische Aktivität von Streptokinaseaktiviertem Plasmin wesentlich stärker gehemmt wird als die esterolytische. Aktivität (37). Um die Beeinflußbarkeit des Fibrinolysesystems durch unser Inhibitormaterial beurteilen zu können, führen wir zur Zeit Versuche durch, die die proteolytische Inhibitoraktivität erfassen. Eine Beeinflussung des thrombotischen Systems scheint uns unwahrscheinlich zu sein, und die uns vorliegenden Inhibitoraktivitäten gegenüber Thrombin sind möglicherweise auf die Ungenauigkeit des Testsystems zurückzuführen.

\section{Danksagung}

Der Deutschen Forschungsgemeinschaft danken wir für die freundliche Unterstützung dieser Arbeit. Ebenfalls möchten wir der NOVO Industrie, Mainz, und der Bayer AG, Wuppertal, für ihre wertvolle Hilfe danken. Frau Priv. Doz. G. HartmannSchröder, Universität Hamburg, sei vielmals gedankt für ihre Beratung bei der Bestimmung des Polychaeten.

\section{Literatur}

1. Markwardt, F. (1957), Hoppe-Seyler's Z. Physiol. Chem. $308,147-156$.

2. Fritz, H., Gebhardt, M., Meister, R. \& Funk, E. (1971) in Proceedings of the International Research Conference on Proteinase Inhibitors (Fritz, H. \& Tschesche, H., eds.), p. 271-280, Walter de Gruyter, Berlin.

3. Frenzel, G. (1926), duBois Archiv 157, 293-314.

4. Weinland, E. (1903), Z. Biol. 44, 1-15.

5. Peanasky, R. J. \& Abu-Erreish, G. M. (1971), in 1.c. (2), p. 281-293.

6. Peanasky, R. J. \& Laskowski, M. Sr. (1960), Biochim. Biophys. Acta 37, 167-169.

7. Rhodes, M. B., Marsh, C. L. \& Kelley, G. W. Jr. (1963), Exp. Parasitol. 13, 266-272.

8. Fraefel, W. \& Acher, A. (1968), Biochim. Biophys. Acta $154,615-617$.

9. Peanasky, R. J., Abu-Erreish, G. M., Gaush, C. R., Hamandberg. G. A., O'Heeran, D., Linkenheit, R. K. \& Kneich, U. (1974) in Bayer-Symposium V., Proteinase Inhibitors (Fritz, H., Tschesche, H., Greene, L. J. \& Truscheit, E. Eds), Springer-Verlag Berlin, Heidelberg, New York.

10. Werle, E., Appel, W. \& Hopp, E. (1958), Naunyn-Schmiedeberg's Arch. Pharmakol. Exper. Pathol. 234, 364-372.

11. Kareem, A. (1973), persönliche $\Lambda^{*}$ itteilung.

12. Cheesman, D. F. (1958), Proceedings Royal Soc. B 149 , 571-587.

13. Norden, D. (1972), Comp. Biochem. Physiol. 42b, 569-576.

14. Uhlenbruck, G., Sprenger, I. \& Ishiyama, I. (1971), diese Z. $9,361-362$.

15. Uhlenbruck, G., Sprenger, I. \& Hermann, G. (1971), diese Z. 9, 494-496.
16. Tschesche, H. \& Dietl, T. (1972), Europ. J. Biochem. 30, $560-570$.

17. Tsschesche, H. (1974), Angew. Chem. 86, 21-40.

18. Tschesche, H. \& Dietl, T (1974), in l.c. (9), p. 586=593.

19. Tschesche, H. \& Rücker, V. A. (1973), Hoppe-Seyler's Z. Physiol. Chem. 354, 1447-1461.

20. Béress, C., Kostmann, H. \& Fritz, H. (1971), Hoppe-Seyler's Z. Physiol. Chem. 353, 111-112.

21. Fritz, H., Brey, B. \& Béress, C. (1972), Hoppe-Seyler's Z. Physiol. Chem. 353, 19-30.

22. Prokop, O., Schlesinger, D. \& Rackwitz, A. (1965), Z. Immunforsch. 129, 402-412.

23. Lowry, O. H., Rosenbrough, N. J., Farr, A. L. \& Randall, R. J. (1951), J. Biol. Chem. 193, 265-275.

24. Fritz, H., Trautschold, I. \& Werle, E. (1974), in Methoden der Enzymatischen Analyse (Bergmeyer, H., ed), Verlag Chemie, S. 1105-1122.

25. Fritz, H., Janmann, E., Meister, R., Pasquay, P., Hochstrasser, K. \& Funk, E. (1971) in 1.c. (2), S. 257-270.

26. Trautschold, I., Werle, E. \& Schweitzer, G. (1974), in Methoden der Enzymatischen Analyse (Bergmeyer, H., ed.) Verlag Chemie, S. 1071-1080.

27. Heimburger, N. \& Schwick, G. (1962), Thrombos. Diathes. Haemorrh. 7, 432-443.

28. Andrews, P. (1965), Biochem. J. 96, 595-606.

29. Felgenhauer, K. (1970) in Protides of the Biological Fluids. Proceedings of the 17th Colloquium, (Peeters, H. ed.) S. 505-509, Bruges 1969.

30. Koide, J. \& Ikenaka, T. (1973), Europ. J. Biochem. 32, 401-407. 
31. Kunitz, M. (1947), J. Gen. Phÿsiol. 30, 291-310.

32. Finkenstadt, W. R., Hemid, M. A., Mattis, J. A., Schrode, J., Sealock, R. W., Wang, D. \& Laskowski, M. S. Jr (1974) in: 1.c. (9), S. 389-411.

33. Kunitz, M. (1948), J. Gen. Physiol. 32, 241-248.

34. Uhlenbruck, G. \& Weis, A. (1973), in Blutgruppenkunde (Urbaschek, B., ed.), S. 65-79, Med. Verlagsgesellschaft.
35. Feeney, R. E. (1971), in: 1.c. (2), S. 189-195.

36. Marx, R., Clemente, P., Werle, E. \& Appel, W. (1959), Blut 5, 267-375.

37. Markus, G. \& Workheiser, W. C. (1962), Fed. Proc. 21, 64-71.

Dr. C. Gauwerky,

Prof. Dr. G. Uhlenbruck

Kerpener Str. 15

D-5000 Köln 41 
